\title{
Establishment and biological characteristics of acquired gefitinib resistance in cell line NCI-H1975/gefinitib-resistant with epidermal growth factor receptor T790M mutation
}

\author{
BAO-XIA ZHAO ${ }^{1,2}$, JING WANG $^{1}$, BO SONG $^{3}$, HONG WEI $^{1}$, WEI-PENG LV ${ }^{1}$, \\ LI-MIN TIAN ${ }^{1}$, MEI LI $^{1}$ and SHEN LV ${ }^{1}$ \\ ${ }^{1}$ Laboratory Center, The Second Affiliated Hospital of Dalian Medical University, Dalian, Liaoning 116027; \\ ${ }^{2}$ College of Medical Laboratory; ${ }^{3}$ Department of Pathology, College of Basic Medical Sciences, \\ Dalian Medical University, Dalian, Liaoning 116004, P.R. China
}

Received January 28, 2014; Accepted November 3, 2014

DOI: $10.3892 / \mathrm{mmr} .2014 .3058$

\begin{abstract}
Non-small cell lung cancer (NSCLC) cells harboring mutations in the epidermal growth factor receptor (EGFR) gene initially respond well to EGFR tyrosine kinase inhibitors (TKI), including gefitinib. However the tumor cells will invariably develop acquired resistance to the drug. The EGFR T790M mutation is generally considered to be the molecular genetic basis of acquired TKI resistance. The present study aimed to explore how the T790M mutation induces tumor cells to escape inhibition by TKI treatment. An acquired gefitinib-resistant cell line (NCI-H1975/GR) was generated from the NCI-H1975 human NSCLC cell line, which harbors the sensitive L858R and resistant T790M mutations of EGFR. The resistant cell line was established by exposing the cells intermittently to increasing concentrations of gefitinib. The mechanisms by which NSCLC acquires resistance to TKIs based on the T790M mutation, were investigated by detecting the protein expression levels of the EGFR/Kirsten rat sarcoma viral oncogene homolog (KRAS)/v-Raf murine sarcoma viral oncogene homolog B (BRAF) transduction pathway, and epithelial-mesenchymal transition (EMT) with immunocytochemistry. The resistance of the NCI-H1975/GR cells to gefitinib was 2.009-fold, as compared with the parent cells; however, the protein expression levels of EGFR, KRAS and BRAF were lower in the resistant cells. Some mesenchymal morphology was observed in the NCI-H1975/GR cells, alongside a decreasing E-cadherin expression and increasing vimentin expression. These results suggest that the reactivation
\end{abstract}

Correspondence to: Professor Shen Lv, Laboratory Center, The Second Affiliated Hospital of Dalian Medical University, 467 Zhongshan Road, Dalian, Liaoning 116027, P.R. China E-mail: lu.shen1956@aliyun.com

Key words: epidermal growth factor receptor, non-small cell lung cancer, tyrosine kinase inhibitors, resistance, epithelial-mesenchymal transition of the EGFR/KRAS/BRAF transduction pathway was not detected in the NCI-H1975/GR cells. EMT may have an important role in the development of acquired resistance to EGFR-TKIs in NSCLC cells with sensitivity and resistance mutations.

\section{Introduction}

Non-small cell lung cancer (NSCLC) is one of the most prevalent malignancies associated with morbidity and mortality worldwide (1). The main therapeutic method used to treat NSCLC is surgical resection. However, when initially diagnosed with NSCLC, $>50 \%$ of patients will already be in the advanced stage, and some may have missed the opportunity for surgery. Furthermore, the patients eligible for surgery will often also require adjuvant chemotherapy. Therefore, chemotherapy has become the widest used clinical approach in the treatment of NSCLC. Unfortunately, traditional chemotherapy has numerous clinical limitations, due to its poor specificity and severe side effects. In order to overcome the shortcomings of traditional chemotherapy, individualized treatment has recently been extensively used. In recent years, molecular targeted drugs have been popularized in individualized cancer treatment, due to their improved specificity and reduced side effects. Epidermal growth factor receptor tyrosine-kinase inhibitors (EGFR-TKIs), such as gefitinib and erlotinib, are the most common molecular targeted drugs used to treat NSCLC. Previous studies have shown that NSCLC cells which harbor activating EGFR mutations, such as exon 19 deletion and the exon 21 missense mutation (L858R), will be sensitive to EGFR-TKIs (2-4). Unfortunately, the majority of patients with NSCLC who are initially sensitive to EGFR-TKIs, will ultimately develop acquired resistance to the drug. Therefore, exploring the mechanisms behind the acquired drug resistance of NSCLC to EGFR-TKI has become an urgent clinical problem.

Some mechanisms regarding the acquired resistance of NSCLC to EGFR-TKIs, have been reported as follows: Secondary mutations, such as EGFR T790M exon 20; encoding gene mutations of Kirsten rat sarcoma viral 
oncogene homolog (KRAS), v-Raf murine sarcoma viral oncogene homolog B (BRAF), mitogen-activated protein kinase and phosphoinositide-3 kinase (PI3K) in the EGFR, downstream of signal transduction; gene amplification of other signal transduction pathways, such as MET; and epithelial-mesenchymal transition (EMT) (5-13). The T790M mutation occurs in $\sim 50 \%$ of NSCLC patients who have developed acquired drug resistance to EGFR-TKIs. Therefore, the EGFR T790M mutation has been generally considered as the molecular genetic basis of TKI-acquired drug resistance. However, it remains unclear how the cells, having harbored the T790M mutation, develop acquired drug resistance. The establishment of an acquired gefitinib resistant subline from an NSCLC cell line, harboring sensitive (exon 21; L858R) and resistant (exon 20; T790M) mutations of EGFR may be helpful for exploring the problem of acquired drug resistance to EGFR-TKIs. The NCI-H1975 human NSCLC cell line was established in 1988, prior to the clinical use of TKIs, and harbors the L858R and T790M double mutations (6). This cell line should initially be sensitive and easily develop acquired resistance to TKIs, following TKI stimulation. Therefore, the NCI-H1975 cell line is an ideal cell line to use for the study of TKI-acquired resistance based on the T790M mutation.

It was previously reported that tumor cells with exon 21 mutations (L858R) or exon 19 deletions in EGFR, showed higher tyrosine kinase activity (2). EGFR-TKIs can suppress the higher tyrosine kinase activity due to these mutations, and block signal transduction from EGFR (14-16). The T790M mutation substitutes methionine for threonine in the 'gatekeeper' region of EGFR, and the bulkier methionine prevents the EGFR-TKIs from binding the ATP pocket of EGFR tyrosine kinase (5). Some studies have suggested that the T790M mutation most likely causes acquired drug resistance by enhancing the ATP affinity of EGFR L858R, and thus reducing the efficacy of the ATP-competitive TKIs (17). These studies indicate that the T790M mutation may cause the cells harboring the EGFR activating mutations, such as L858R, to maintain the tyrosine kinase activity of EGFR, and subsequently lose sensitivity to EGFR-TKIs. However, it remains unclear how the T790M mutation induces tumor cells that were initially sensitive to EGFR-TKIs, to escape from the inhibition of the drug. The present study developed an acquired gefitinib-resistant cell line (NCI-H1975/GR) from the NCI-H1975 cell line. Furthermore, by detecting the protein expression levels of the EGFR/KRAS/BRAF transduction pathway, and observing the EMT in the process of acquired gefitinib resistance development, the possible mechanisms by which the T790M mutation induces NSCLC to develop acquired resistance to TKIs were investigated.

\section{Materials and methods}

Drugs and cell line. Gefitinib powder was purchased from Selleckchem (Radnor, PA, USA). The NCI-H1975 human NSCLC cell line was purchased from the Cell Culture Center of the Institute of Basic Medical Sciences, Chinese Academy of Medical School (Beijing, China). The cells were cultured in RPMI-1640 medium supplemented with $10 \%$ fetal calf serum, $100 \mathrm{U} / \mathrm{ml}$ penicillin and $100 \mu \mathrm{g} / \mathrm{ml}$ streptomycin (Gibco-BRL,
Grand Island, NY, USA), and maintained in a $5 \% \mathrm{CO}_{2}$ humidified incubator at $37^{\circ} \mathrm{C}$.

Establishment of an acquired gefitinib-resistant cell line NCI-H1975/GR. To develop the acquired gefitinib-resistant cell line, the NCI-H1975 cells were initially exposed to $12 \mu \mathrm{mol} / \mathrm{l}$ gefitinib for $24 \mathrm{~h}$. The surviving cells were washed with RPMI-1640, and cultured in the drug-free medium until they had reached $80 \%$ confluence. The cells were further exposed to $12 \mu \mathrm{mol} / 1$ gefitinib for $24 \mathrm{~h}$. This process was repeated with increasing drug concentrations, until $80 \mu \mathrm{mol} / 1$. After the cells had been cultured in drug-free medium for two weeks, the subsequent investigations were conducted.

Growth inhibition assay. An MTT assay and the trypan blue dye exclusion method were used to measure cell sensitivity to gefitinib. The MTT assay was performed according to the following protocol. The parent and resistant cells, growing exponentially, were harvested and seeded into 96-well plates at a density of $5 \times 10^{3}$ cells/well overnight. The cells were treated with gefitinib at the indicated doses for $72 \mathrm{~h}$ at $37^{\circ} \mathrm{C}$, after which $20 \mu 1$ MTT solution [Sigma-Aldrich, St Louis, MO, USA; $5 \mathrm{mg} / \mathrm{ml}$ in phosphate-buffered saline (PBS)] was added to each well, and the plates were incubated for $4 \mathrm{~h}$ at $37^{\circ} \mathrm{C}$. The plates were then centrifuged at 2,250 $\mathrm{x}$ g for $10 \mathrm{~min}$, the medium was aspirated from each well and $150 \mu 1$ dimethyl sulfoxide was added to each well, in order to dissolve the formazan crystals. The optical density was measured at a wavelength of $492 \mathrm{~nm}$ using an automatic microplate reader (Thermo Labsystems, Helsinki, Finland). Absorbance values were expressed as a percentage of that for untreated cells, and the half maximal inhibitory concentration of gefitinib $\left(\mathrm{IC}_{50}\right)$ was calculated. In addition, the number of viable cells from the parent and resistant cell lines were determined by the trypan blue (Spectrum Chemicals \& Laboratory Products, Shanghai, China) dye exclusion method, and the number of viable cells were counted using a hemocytometer (Shanghai Qiujing Biochemical Reagent and Instrument Co., Ltd., Shanghai, China) every $24 \mathrm{~h}$ for 4 days. Each assay was performed in triplicate.

Apoptosis assay. The percentage of apoptotic parent and resistant cells, with or without gefitinib stimulation, were determined using Annexin V-fluorescein isothiocyanate (FITC) and propidium iodide (PI) staining (Biouniquer, China). The cells were treated with or without gefitinib $(20 \mu \mathrm{mol} / \mathrm{l})$ for $24 \mathrm{~h}$, harvested by trypsin (Sigma-Aldrich) digestion, washed twice with PBS, and then suspended in $500 \mu 1$ Annexin V Binding buffer. Thereafter, $5 \mu \mathrm{l}$ Annexin V-FITC and $5 \mu \mathrm{l}$ PI were added to the samples, which were incubated for $10 \mathrm{~min}$ at room temperature in the dark, according to the manufacturer's instructions. The apoptotic cells were detected using a FACSCalibur ${ }^{\mathrm{TM}}$ flow cytometer (BD Biosciences, Franklin Lakes, NJ, USA).

Morphological analysis. Changes to the morphology of the parent and gefitinib-resistant cells, in response to gefitinib treatment, including size, shape and development of pseudopodia, were directly observed under an inverted microscope (Olympus Corporation, Tokyo, Japan). 
Table I. Primers of EGFR, KRAS and BRAF.

Primer

Sequence (5'-3')

Exon 18 of EGFR

Exon 19 of EGFR

5'-GCTTGTGGAGCCTCTTACA-3'

5'-GCCAGGGACCTTACCTTAT-3'

5'-TGGATCCCAGAAGGTGAGAA-3'

5'-AGCAGAAACTCACATCGAGGA-3'

Exon 20 of EGFR

5'-ACTGACGTGCCTCTCCCTC-3'

5'-CCCGTATCTCCCTTCCCTG-3'

Exon 21 of EGFR

5'-CGCAGCATGTCAAGATCA-3'

5'-CCTCCTTACTTTGCCTCC-3'

Exon 2 of KRAS

5'-AGGCCTGCTGAAAATGACT-3'

5'-AATGGTCCTGCACCAGTAA-3'

Exon 15 of BRAF

5'-CTCTTCATAATGCTTGCTCTGATAGG-3'

5'-TAGTAACTCAGCAGCATCTCAGG-3'

EGFR, epidermal growth factor receptor; KRAS, Kirsten rat sarcoma viral oncogene homolog; BRAF, v-Raf murine sarcoma viral oncogene homolog B.

Cell proliferation assay. The cells, growing exponentially, were harvested and seeded into 24 -well plates at a density of $1.5 \times 10^{4}$ cells/well. The parent and resistant cells were counted with a hematocytometer every $24 \mathrm{~h}$ for 7 days. The proliferation curves were charted, and the cell population doubling times were calculated using the following equation: $\mathrm{T}=\operatorname{tg}^{2 /(\operatorname{lgNt}-\operatorname{lgN} 0)}(\mathrm{T}$, population doubling time; $\mathrm{t}$, continuous culture time; $\mathrm{N}_{\mathrm{t}}$, terminal number of cells; $\mathrm{N}_{0}$, initial number of cells. Time units in $h ; \lg , \log _{10}$ ).

Cell cycle analysis. The cell cycle distributions of the parent and resistant cells were analyzed by flow cytometry. The parent and resistant cells were treated with or without gefitinib $(20 \mu \mathrm{mol} / \mathrm{l})$ for $24 \mathrm{~h}$. The cells were then harvested by trypsin digestion, washed twice with ice-cold PBS, fixed in $70 \%$ ethanol and then maintained at $4^{\circ} \mathrm{C}$ overnight. Following the removal of ethanol by centrifugation, the cells were washed twice with PBS and stained with PI/RNase solution for $30 \mathrm{~min}$ in a $37^{\circ} \mathrm{C}$ water bath, according to the manufacturer's instructions(Beyotime Institute of Biotechnology, Haimen, China). Cell cycle distribution was detected using a FACSCalibur $^{\mathrm{TM}}$ flow cytometer, and the data were analyzed using Cellquest ${ }^{\mathrm{TM}}$ (BD Biosciences).

Polymerase chain reaction-high resolution melting analysis (PCR-HRMA). Mutation analysis of exons 18-21 of EGFR, exon 2 of KRAS and exon 15 of BRAF gene was performed using PCR-HRMA (LightScanner ${ }^{\circledR}$ HRI 96; Biofire Diagnostics, Inc., Salt Lake City, UT, USA). Genomic DNA was extracted from the parent and resistant cells using a Genomic DNA Extraction kit (TIANGEN Biotech Co., Ltd., Beijing, China), and exons 18-21 of EGFR, exon 2 of KRAS and exon 15 of BRAF were amplified by PCR. The PCR reaction mixture contained 10X PCR buffer (Takara Biotechnology Co., Ltd., Dalian, China ), $2.5 \mathrm{mmol} / \mathrm{l} \mathrm{dNTPs,} 25 \mathrm{mmol} / 1 \mathrm{MgCl}_{2}, 100 \mu \mathrm{M}$ primer, $5 \mathrm{U} / \mu 1$ HotStart Taq (Takara Biotechnology Co., Ltd., Dalian, China), 5 ng genomic DNA and 10X LC Green Plus (Biochem, Salt Lake City, UT, USA). The primer sequences are shown in Table I. PCR amplification conditions were set as follows: 1) EGFR exons 18/19: $95^{\circ} \mathrm{C}$ for $5 \mathrm{~min}$; 45 cycles of $95^{\circ} \mathrm{C}$ for $15 \mathrm{~s}, 60^{\circ} \mathrm{C}$ for $1 \mathrm{~min}$; 2) EGFR exon $20 / 21$ and KRAS exon 2: $95^{\circ} \mathrm{C}$ for $10 \mathrm{~min} ; 45$ cycles of $95^{\circ} \mathrm{C}$ for $30 \mathrm{~s}, 54^{\circ} \mathrm{C}$ for $10 \mathrm{~s}$ and $72^{\circ} \mathrm{C}$ for $1 \mathrm{~min}$; 3) BRAF exon 15 : $95^{\circ} \mathrm{C}$ for $10 \mathrm{~min}$; 45 cycles of $95^{\circ} \mathrm{C}$ for $30 \mathrm{~s}, 56^{\circ} \mathrm{C}$ for $10 \mathrm{~s}$ and $72^{\circ} \mathrm{C}$ for $30 \mathrm{~s}$. The PCR products and melting curves were analyzed using the LightScanner ${ }^{\circledR}$ software Call-IT (version 1.5), according to the manufacturer's instructions.

Western blot analysis. The primary antibodies used for western blot analysis were as follows: Anti-EGFR (mouse monoclonal antibody, Thermo Fisher Scientific, Waltham, MA, USA), anti-pEGFR (mouse monoclonal antibody; Try1068; Cell Signaling Technology, Danvers, MA, USA), anti-RAS (mouse monoclonal antibody; Abcam, Cambridge, UK); anti-RAF (mouse monoclonal antibody; Santa Cruz Biotechnology Inc., Dallas, TX, USA) and anti- $\beta$-actin (mouse monoclonal antibody; Beijing Zhongshan Golden Bridge Biotechnology, Beijing, China). Antibodies were diluted to $1: 50,1: 1,000,1: 20,1: 500$ and 1:500, respectively. Whole-cell extracts from the parent and resistant cells were prepared using a Total Protein Extraction kit (Nanjing KeyGen Biotech Co., Ltd., Nanjing, China). The protein concentrations were determined using the Bicinchoninc Acid Protein Assay kit (Beyotime Institute of Biotechnology, Haimen, China). Equal amounts of protein $(100 \mu \mathrm{g})$ were separated by $10 \%$ SDS-PAGE and transferred to polyvinylidene difluoride membranes (EMD Millipore, Billerica, MA, USA). The membranes were then blocked with $5 \%$ skim milk or $3 \%$ bovine serum albumin, and incubated at $4^{\circ} \mathrm{C}$ overnight (anti-pEGFR, anti-RAS, anti-RAF and anti- $\beta$-actin) or $37^{\circ} \mathrm{C}$ for $2 \mathrm{~h}$ (anti-EGFR) with the primary antibodies, according to the manufacturer's instructions. Subsequent to washing with Tris-buffered saline with Tween ${ }^{\circledR}$ (TBST) three times, the membranes were incubated with horseradish peroxidase-conjugated goat anti-mouse immunoglobulin $\mathrm{G}$ secondary antibodies (1:10,000 dilution; 
Beijing Zhongshan Golden Bridge Biotechnology) for $1 \mathrm{~h}$ at room temperature. The membranes were washed a further three times with TBST, and the blots were visualized using an Enhanced Chemiluminescence kit (Beyotime Institute of Biotechnology). The bands were analyzed using Gel-Pro ${ }^{\circledR}$ Analyzer software (Media Cybernetics, Rockville, MD, USA).

Immunocytochemistry. Anti-E-cadherin and anti-vimentin were purchased from Beijing Zhonghshan Golden Bridge Biotechnology. The parent and resistant cells, having grown to $80 \%$ confluence, were fixed in a chamber slide with cold acetone for $10 \mathrm{~min}$ and washed three times with PBS. The slides were incubated with $3 \% \mathrm{H}_{2} \mathrm{O}_{2}$ for $20 \mathrm{~min}$. The cells were then washed a further three times with PBS and blocked with goat serum for $15 \mathrm{~min}$. The excess serum was poured off of the slides, and the slides were incubated with the primary antibodies overnight at $4^{\circ} \mathrm{C}$, according to the manufacturer's instructions. Following the incubation, the cells were washed three times with PBS and incubated with biotin-conjugated secondary antibody (SP9002, monoclonal goat anti-mouse IgG; Beijing Zhonghshan Golden Bridge Biotechnology) at $37^{\circ} \mathrm{C}$ for $30 \mathrm{~min}$. The cells were washed again with PBS three times and incubated with horseradish peroxidase at $37^{\circ} \mathrm{C}$ for $30 \mathrm{~min}$. Following another three washes the cells were stained with diaminobenzidine (Beijing Zhongshan Golden Bridge Biotechnology). The cell nuclei were stained with hematoxylin (Beyotime Institute of Biotechnology) for 1-2 min and washed with water for $10 \mathrm{~min}$, to return to blue. Positive expression was indicated by a yellow cell membrane or cytoplasm, and the localization of positive expression was recorded. The positive expression intensities of the resistant cells were compared to that of the parent cells in the corresponding expression localizations

Statistical analysis. All of the assays were repeated $\geq 3$ times. An independent sample t-test and a one-way analysis of variance were used to determine the statistical significance of the mean differences between the groups. $\mathrm{P}<0.05$ was considered to indicate a statistically significant difference.

\section{Results}

Growth inhibition assay. Over eight months, the acquired gefitinib-resistant cell line, generated from the NCI-H1975 NSCLC cell line, was established and named NCI-H1975/GR. The resistance index of NCI-H1975/GR was 2.009 and the $\mathrm{IC}_{50}$ was $12.343 \mu \mathrm{mol} / 1$, which was markedly higher as compared with the parent NCI-H1975 cells $(6.145 \mu \mathrm{mol} / \mathrm{l})$ (Fig. 1A). The number of viable NCI-H1975/GR cells treated with gefitinib at various concentrations decreased on day 1 , as did those of the parent cells. However, the number of viable NCI-H1975GR cells reached a stable level and did not continuously decrease between days 2-4 (Fig. 1B). Furthermore, the number of viable NCI-H1975/GR cells treated with gefitinib at the indicated concentrations were higher, as compared with the parent cells.

Apoptosis assay. The percentage of apoptotic NCI-H1975GR cells $(4.45 \pm 0.14 \%)$ was significantly higher, as compared with
A

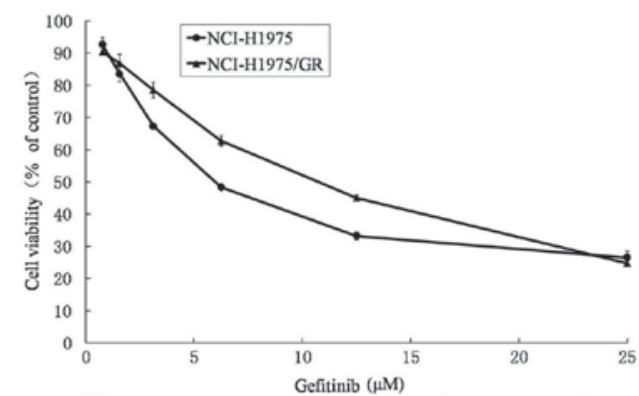

B
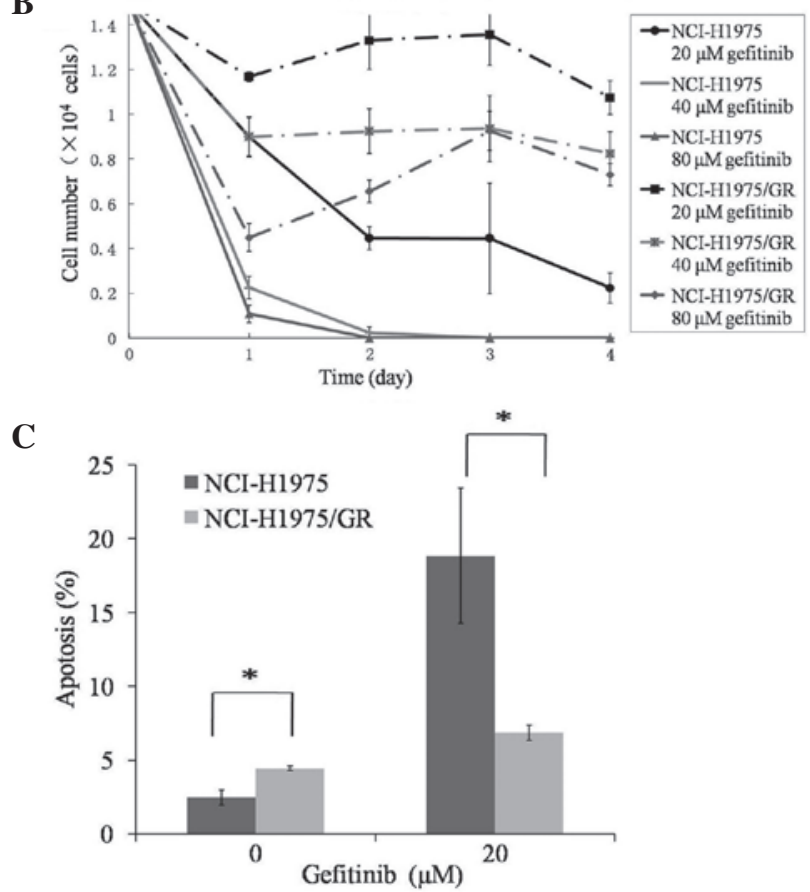

Figure 1. Assessment of the gefitinib resistance of the NCI-H1975/GR human non-small cell lung cancer cell line. (A) Cell viability of the parent NCI-H1975 and NCI-H1975/GR cell lines treated with gefitinib, at the indicated concentrations for $72 \mathrm{~h}$, as determined by MTT assay. (B) The number of viable NCI-H1975 and NCI-H1975/GR cells, treated with gefitinib at concentrations of 20,40 and $80 \mu \mathrm{mol} / 1$, was determined by trypan blue dye exclusion method, with the number of cells counted using a hemocytometer every $24 \mathrm{~h}$ for 4 days. (C) The percentage of apoptotic NCI-H1975/GR and NCI-H1975 cells, with or without gefitinib treatment, were detected by Annexin V-fluorescein isiothiocyanate and propidium iodide staining, using flow cytometry. The values represent the mean \pm standard deviation of $>3$ independent experiments. ${ }^{*} \mathrm{P}<0.05$. GR, gefitinib-resistant.

the NCI-H1975 cells $(2.47 \pm 0.51 \%)(\mathrm{P}<0.05)$, in the absence of gefitinib (Fig. 1C). Whereas, the percentage of apoptotic NCI-H1975/GR cells was significantly lower, as compared with the parent cells, in response to $20 \mu \mathrm{mol} / 1$ gefitinib treatment $(\mathrm{P}<0.05)$.

Morphological analysis. Notable morphological differences between the NCI-H1975/GR and NCI-H1975 cells were observed. The NCI-H1975/GR cells acquired an oval shape from the long spindle shape of the parent cells. Furthermore, the resistant cells were smaller, as compared with the parent cells, and some developed pseudopodia (Fig. 2).

Cell proliferation assay. Cell proliferation curves were charted for the parent and gefitinib-resistant cells. The cell population doubling time of the NCI-H1975/GR cells was $46.535 \pm 0.428 \mathrm{~h}$, 


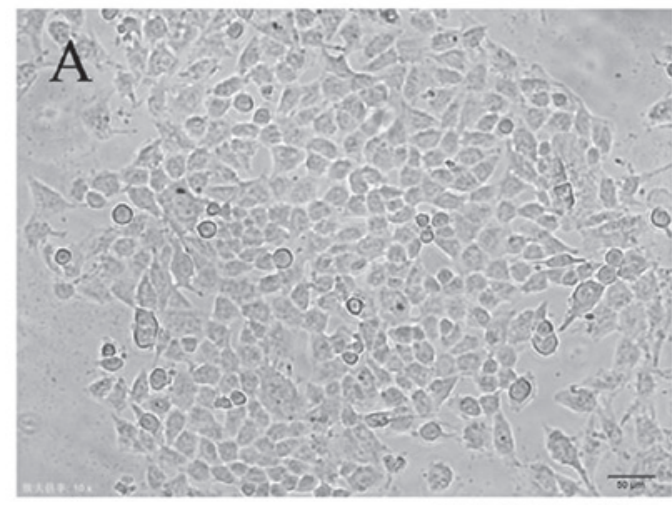

NCI-H1975/GR

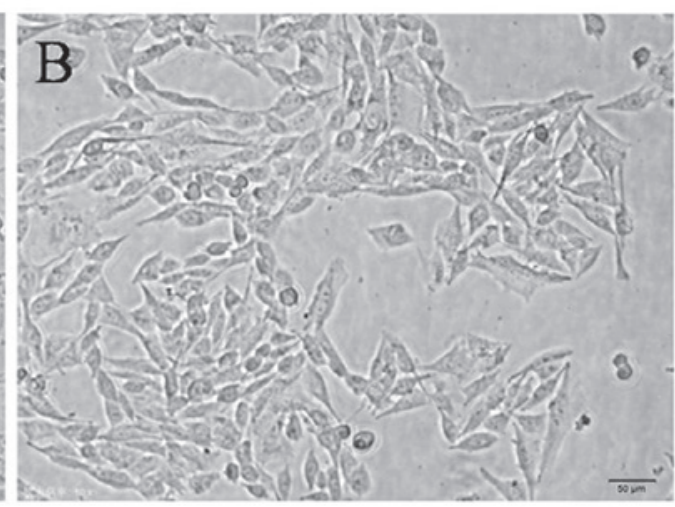

NCI-H1975

Figure 2. Morphological differences observed between the NCI-H1975/GR (gefitinib-resistant) and NCI-H1975 parent human non-small cell lung cancer cells The majority of (A) NCI-H1975/GR cells were oval-shaped and (B) NCI-H1975 cells were long spindle-shaped.

A

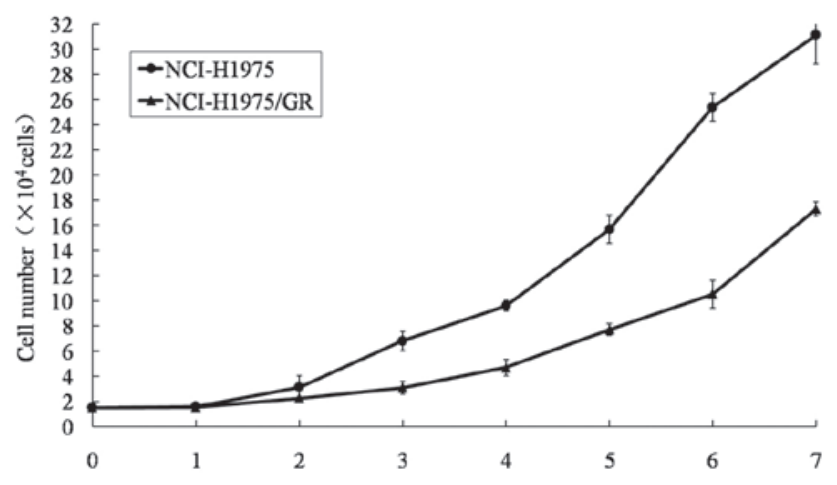

B

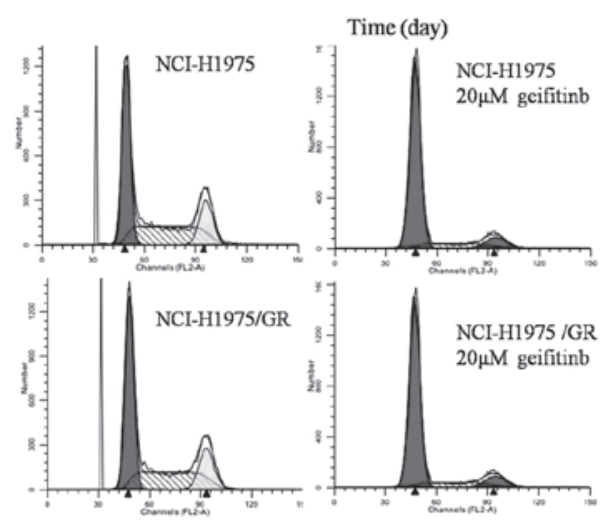

C

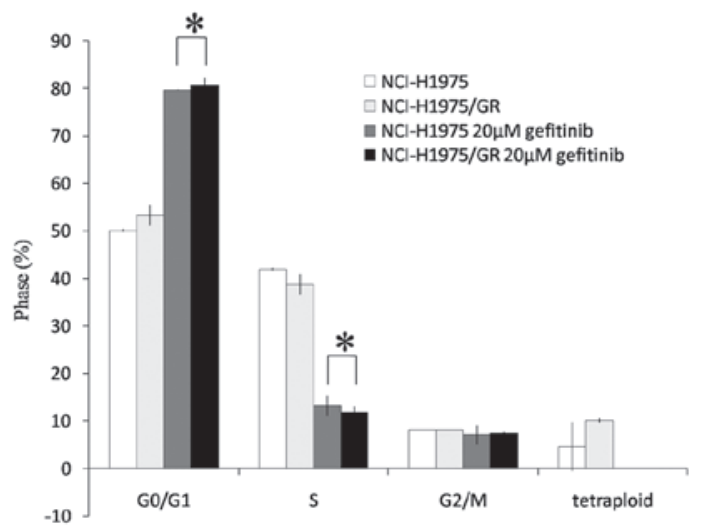

Figure 3. Assessment of the growth of the NCI-H1975/GR (gefitinib-resistant) human non-small cell lung cancer cell line. (A) Proliferation curves of the NCI-H1975/GR cells and NCI-H1975 parent cells were charted by counting the number of viable cells with a hemocytometer every $24 \mathrm{~h}$ for seven days. (B) Cell cycle distributions of the NCI-H1975/GR and NCI-H1975 cells, with or without gefitinib treatment for $24 \mathrm{~h}$, as detected by flow cytometry. (C) Quantification of cell cycle distributions of the NCI-H1975/GR and NCI-H1975 cells. The values represent the mean \pm standard deviation of $>3$ independent experiments. ${ }^{*} \mathrm{P}<0.05$.

which was $16.004 \pm 1.426 \mathrm{~h}$ longer as compared with the NCI-H1975 cells ( $30.531 \pm 1.823$ h) $(\mathrm{P}<0.05$; Fig. $3 \mathrm{~A})$.

Cell cycle analysis. The proportion of NCI-H1975/GR cells within the $\mathrm{G}_{0} / \mathrm{G}_{1}$ phase was slightly higher, as compared with the NCI-H1975 cells, and the proportion of tetraploid cells was also slightly higher $(\mathrm{P}>0.05)$. Following treatment with gefi- tinib $(20 \mu \mathrm{mol} / \mathrm{l})$ for $24 \mathrm{~h}$, the proportion of NCI-H1975/GR cells within the $G_{0} / G_{1}$ phase was markedly increased and within the $\mathrm{S}$ phase decreased (Fig. 3B and C). Furthermore, the tetraploid cells disappeared following gefitinib treatment.

Mutation analysis of EGFR, KRAS and BRAF. With the exception of T790M and L858R, no novel mutations were observed 
A NCI-H1975 NCI-H1975/GR B
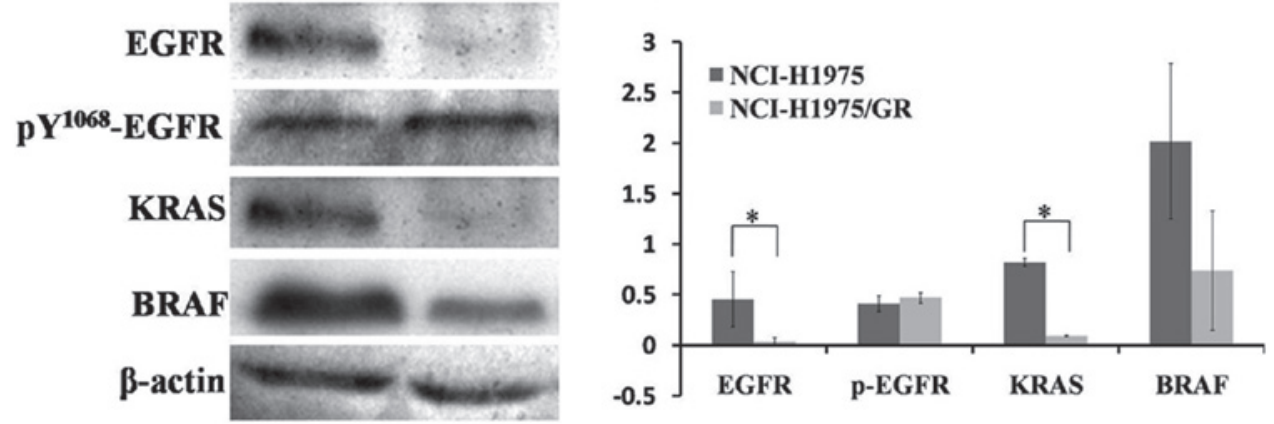

Figure 4. The protein expression levels of the EGFR/KRAS/BRAF transduction pathway. (A) Protein expression levels of EGFR, pY1068-EGFR, KRAS and BRAF in the NCI-H1975/GR and NCI-H1975 parent human non-small cell lung cancer cells, as determined by western blotting. $\beta$-actin was used as an internal control. (B) The relative signal intensities of proteins to $\beta$-actin in the NCI-H1975/GR and NCI-H1975 cells, as quantified by Gel-Pro ${ }^{\circledR}$ analyzer software. The values represent the mean \pm standard deviation of $>3$ independent experiments. "P $<0.05$. EGFR, epidermal growth factor receptor; GR, gefinitib-resistant; KRAS, Kirsten rat sarcoma viral oncogene homolog; BRAF, v-Raf murine sarcoma viral oncogene homolog B.

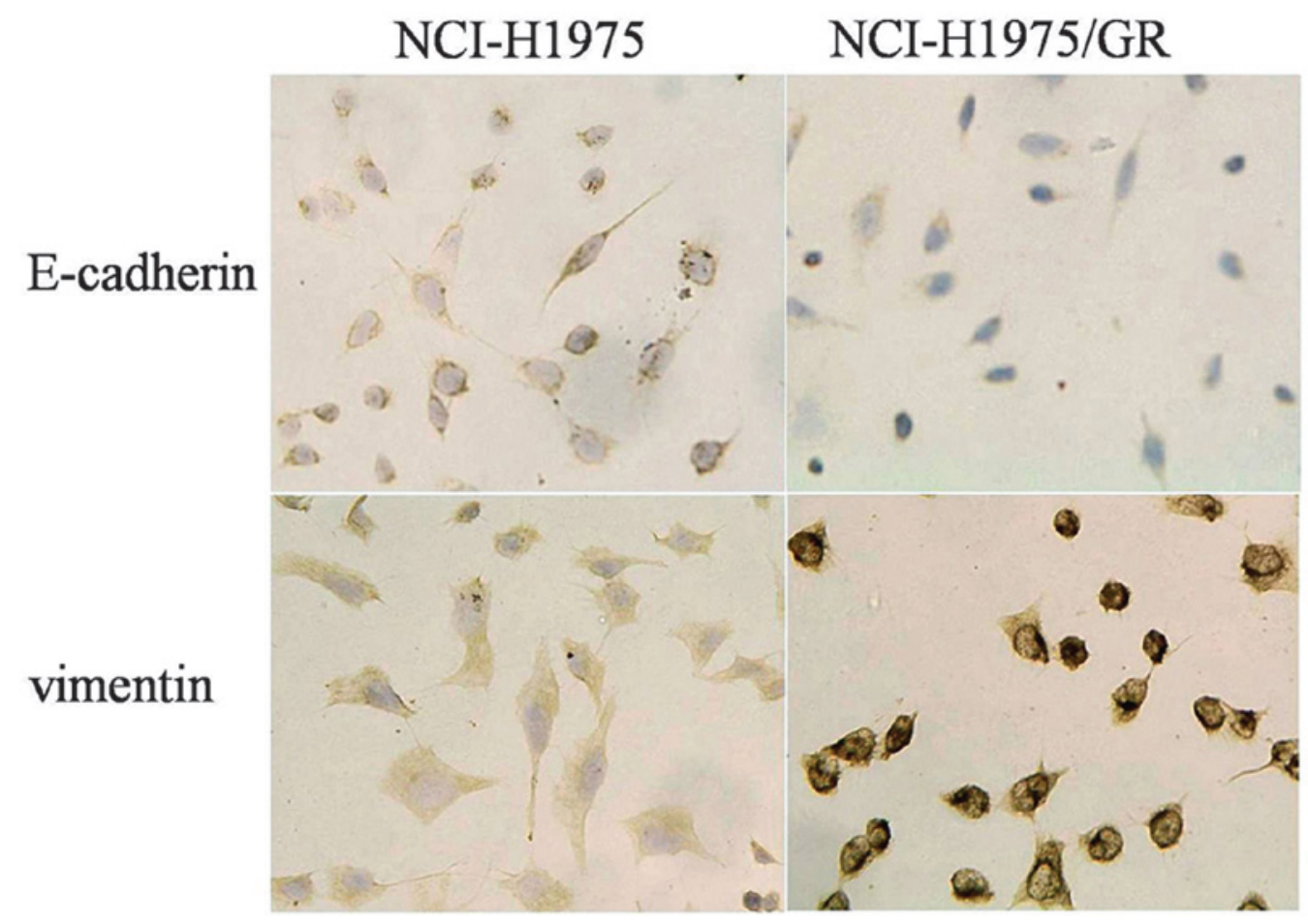

Figure 5. The protein expressions of E-cadherin and vimentin in the NCI-H1975/GR (gefitinib-resistant) and NCI-H1975 parent human non-small cell lung cacer cells, as detected by immunocytochemistry.

in the EGFR, KRAS and BRAF genes of the NCI-H1975/GR cell line, by PCR-HRMA.

Proteinexpressionlevelsofthe EGFR/KRAS/BRAFtransduction pathway. The protein expressions in the EGFR/KRAS/BRAF transduction pathway were detected by western blotting. The protein expression levels in the NCI-H1975/GR cells were lower, as compared with the NCI-H1975 cells; however, the expression levels of pY1068-EGFR protein were slightly higher in the NCI-H1975/GR cells, as compared with the NCI-H1975 cells (Fig. 4). These results indicate that the EGFR/KRAS/BRAF pathway was not re-activated in the development of resistance to EGFR-TKIs.
EMT of NCI-H1975/GR cells, as detected by immunocytochemistry. The expression of E-cadherin was lower, whereas the expression of vimentin was higher in the NCI-H1975/GR cells, as compared with the NCI-H1975 cells (Fig. 5).

\section{Discussion}

The majority of cases of NSCLC which harbor activating EGFR mutations, such as exon 19 deletion and exon 21 mutation (L858R substitution), are initially sensitive to EGFR-TKIs; however, the vast majority of them ultimately acquire resistance to the drug (18-20). Notably, the tumors of $\sim 50 \%$ of patients who develop acquired resistance to 
EGFR-TKIs harbor the exon 20 T790M EGFR mutation. The T790M mutation may develop during the process of acquired resistance to TKIs, but could also be primary, since a small number of patients with NSCLC already harbor the T790M mutation, prior to EGFR-TKI exposure (21-24). The T790M mutation is considered to be the basis by which NSCLC develops acquired resistance to TKIs. Therefore, it is necessary to explore the mechanisms through which the T790M mutation results in the development of resistance to TKIs, for the increasing benefits of patients with NSCLC. The present study developed an acquired gefitinib-resistant cell line from the NCI-H1975 NSCLC cell line, which was considered to be sensitive (harboring L858R mutation) and have the potential to develop resistance to TKIs easily (harboring T790M mutation).

Until now, numerous acquired resistance cell lines have been established. The representative PC-9/ZD was established in 2005 and was the first human NSCLC cell line resistant to gefitinib. It was generated from the PC-9 cell line with an exon 19 deletion of EGFR. PC-9/ZD cells are 182-fold more resistant to gefitinib, as compared with their parent cells. However, there were no significant differences observed between the $\mathrm{PC}-9 / \mathrm{ZD}$ and $\mathrm{PC}-9$ parent cells regarding cell proliferation, microscopic morphology and the DNA sequence of EGFR (25). The H3255 GR was established in 2006 and is another representative NSCLC cell line resistant to EGFR-TKIs. It was developed by prolonged exposure of the gefitinib-sensitive H3255 cell line, with EGFR L858R, to gefitinib. The H3255 GR cells are 100-fold more resistant to gefitinib, as compared with their parental cells. It was demonstrated that the H3255 GR cell line acquired a T790M mutation in a small fraction of the amplified alleles, which was detected by a highly sensitive high performance liquid chromatography-based technique, but not by common direct DNA sequencing (26). The HCC827 GR was established in 2007 and is another NSCLC cell line resistant to EGFR-TKIs, which was also developed by exposure of gefitinib-sensitive HCC 827 cells, with exon 19 deletion of EGFR, to increasing concentrations of gefitinib. The HCC827 GR cells are 100-fold more resistant to gefitinib, as compared with their parental cells. The HCC827 GR cells showed amplification of MET, which caused gefitinib resistance by driving human epidermal growth factor receptor 3-dependent activation of PI3K (27).

The present study used the NCI-H1975 cell line, which is genetically different from the cell lines mentioned above. The NCI-H1975 cell line harbors not only the L858R mutation but also the T790M mutation. In order to develop a gefitinib-resistant cell line, the NCI-H1975 cells were exposed to increasing concentrations of gefitinib. The established gefitinib-resistant cell line, NCI-H1975/GR, was 2.009-fold more resistant to gefitinib, as compared with their parental cells. The percentage of apoptotic NCI-H1975/GR cells decreased, in response to treatment with gefitinib $(20 \mu \mathrm{mol} / \mathrm{l})$, as compared with the NCI-H1975 cells. In addition, the speed of growth of the NCI-H1975/GR cells slowed down (doubling time, $46.535 \pm 0.428 \mathrm{~h}$ ), as compared with the NCI-H1975 cells $(30.531 \pm 1.823 \mathrm{~h})$. These results indicate that the NCI-H1975/GR cell line has low resistance to gefitinib, and that it may be used to explore the mechanisms of TKI-acquired resistance, based on the T790M mutation.
The T790M mutation in the NCI-H1975/GR cells was initially confirmed using PCR-HRMA. The results of the present study indicate that the development of resistance to TKIs was not directly associated with the presence of the T790M mutation. It is well known that NSCLC tumor cells harboring activating mutations in exons 18, 19 and 21 of the EGFR, are sensitive to EGFR-TKIs. These exons were detected by PCR-HRMA in the present study; however, these mutations were not identified, nor the disappearance of the L858R mutation, which had presented in the NCI-H1975 parent cells. The activating mutations of KRAS and BRAF genes have been shown to correlate with primary resistance of NSCLC to TKIs (28). However, these activating and new mutations of exon 2 of KRAS and exon 15 of BRAF were not observed in the present study. These results suggest that the common mutations, which are known to associated with resistance, could not directly result in the development of acquired resistance to TKIs. Generally, the development of acquired resistance to TKIs, based on the T790M mutation, has been attributed to EGFR reactivation, due to drug-binding deficiency $(5,6)$. However, in the present study, the protein expression levels of EGFR, as well as those of KRAS and BRAF, in the NCI-H1975/GR cells were decreased, as compared with the parental cells. These results suggest that the EGFR transduction pathway was not reactivated in the process of acquired resistance in NCI-H1975 cells. Therefore, other mechanisms of resistance to TKIs should be considered.

In 2011, Suda et al (29) established an acquired erlotinib-resistant HCC4006ER NSCLC cell line. The parental cell line harbored the activating mutation of EGFR (exon 19 deletion). In the resistant cell line, no novel mutations of EGFR were detected, whereas some morphological changes associated with EMT were observed, such as loss of intercellular connection and polarity. These findings indicate that EMT was associated with the acquired resistance to TKIs of NSCLC cells harboring a mutation in the EGFR gene. In addition, Rho et al (30) established an acquired gefitinib-resistant A549/GR NSCLC cell line. In this cell line, EMT was also observed. In the present study, some mesenchymal morphologies were detected in the NCI-H1975/GR cells. Furthermore, the EMT in the NCI-H1975/GR cells was examined by detection of the epithelial marker E-cadherin and the mesenchymal marker vimentin. The expression of E-cadherin was lower, whereas the expression of vimentin was higher in the NCI-H1975/GR cells, as compared with the NCI-H1975 cells. These results indicate that EMT may have a role in the development of acquired resistance to EGFR-TKIs in NSCLC cells harboring activating and resistant mutations of EGFR. How EMT promotes the development of acquired resistance in NSCLC cells requires further study.

In conclusion, the present study established an acquired gefitinib-resistant cell line NCI-H1975/GR from the NCI-H1975 cell line, harboring the L858/T790M double mutation. Reactivation of the EGFR/KRAS/BRAF transduction pathway was not observed in the gefitinib-resistant NCI-H1975/GR cells. The results suggested that the EMT may have an important role in the development of acquired resistance to EGFR-TKIs in NSCLC cells with mutations of sensitivity and resistance. 


\section{Acknowledgements}

This study was supported by the National Natural Science Foundation of China (grant no. 81071805).

\section{References}

1. Jemal A, Bray F, Center MM, Ferlay J, Ward E and Forman D: Global cancer statistics. CA Cancer J Clin 61: 69-90, 2011.

2. Lynch TJ, Bell DW, Sordella R, et al: Activating mutations in the epidermal growth factor receptor underlying responsiveness of non-small-cell lung cancer to gefitinib. N Engl J Med 350: 2129-2139, 2004.

3. Paez JG, Jänne PA, Lee JC, et al: EGFR mutations in lung cancer: correlation with clinical response to gefitinib therapy. Science 304: 1497-1500, 2004

4. Pao W, Miller V, Zakowski M, et al: EGF receptor gene mutations are common in lung cancers from 'neversmokers' and are associated with sensitivity of tumors to gefitinib and erlotinib. Proc Natl Acad Sci USA 101: 13306-13311, 2004.

5. Kobayashi S, Boggon TJ, Dayaram T, et al: EGFR mutation and resistance of non-small cell lung cancer to gefitinib. N Engl J Med 352: 786-792, 2005.

6. Pao W, Miller VA, Politi KA, et al: Acquired resistance of lung adenocarcinomas to gefitinib or erlotinib is associated with a second mutation in the EGFR kinase domain. PLoS Med 2: e73, 2005.

7. Ohashi K, Sequist LV, Arcila ME, et al: Lung cancers with acquired resistance to EGFR inhibitors occasionally harbor BRAF gene mutations but lack mutations in KRAS, NRAS, or MEK1. Proc Natl Acad Sci USA 109: E2127-E2133, 2012.

8. Ercan D, Xu C, Yanagita M, et al: Reactivation of ERK signaling causes resistance to EGFR kinase inhibitors. Cancer Discov 2 : 934-947, 2012.

9. Faber AC, Corcoran RB, Ebi $\mathrm{H}$, et al: BIM expression in treatment-naïve cancers predicts responsiveness to kinase inhibitors. Cancer Discov 1: 352-365, 2011.

10. Engelman JA, Zejnullahu K, Mitsudomi T, et al: MET amplification leads to gefitinib resistance in lung cancer by activating ERBB3 signaling. Science 316: 1039-1043, 2007.

11. Bean J, Brennan C, Shih JY, et al: MET amplification occurs with or without T790M mutations in EGFR mutant lung tumors with acquired resistance to gefitinib or erlotinib. Proc Natl Acad Sci USA 104: 20932-20937, 2007.

12. Yauch RL, Januario T, Eberhard DA, et al: Epithelial versus mesenchymal phenotype determines in vitro sensitivity and predicts clinical activity of erlotinib in lung cancer patients. Clin Cancer Res 11: 8686-8698, 2005.

13. Suda K, Tomizawa K, Fujii M, et al: Epithelial to mesenchymal transition in an epidermal growth factor receptor-mutant lung cancer cell line with acquired resistance to erlotinib. J Thorac Oncol 6: 1152-1161, 2011.

14. Mok TS, Wu YL, Thongprasert S, et al: Gefitinib or carboplatin-paclitaxel in pulmonary adenocarcinoma. N Engl J Med 361: 947-957, 2009.
15. Mitsudomi T, Morita S, Yatabe Y, et al: Gefitinib versus cisplatin plus docetaxel in patients with non-small-cell lung cancer harboring mutations of the epidermal growth factor receptor (WJTOG3405): an open label, randomized phase 3 trial. Lancet Oncol 11: 121-128, 2010.

16. Rosell R, Moran T, Queralt C, et al: Screening for epidermal growth factor receptor mutations in lung cancer. N Engl J Med 361: 958-967, 2009.

17. Yun CH, Mengwasser KE, Toms AV, et al: The T790M mutation in EGFR kinase causes drug resistance by increasing the affinity for ATP. Proc Natl Acad Sci USA 105: 2070-2075, 2008.

18. Costa DB, Kobayashi S, Tenen DG and Huberman MS: Pooled analysis of the prospective trials of gefitinib monotherapy for EGFR-mutant non-small cell lung cancers. Lung Cancer 58: 95-103, 2007.

19. Nguyen KS, Kobayashi S and Costa DB: Acquired resistance to epidermal growth factor receptor tyrosine kinase inhibitors in non-small-cell lung cancers dependent on the epidermal growth factor receptor pathway. Clin Lung Cancer 10: 281-289, 2009.

20. Sequist LV, Bell DW, Lynch TJ and Haber DA: Molecular predictors of response to epidermal growth factor receptor antagonists in non-small-cell lung cancer. J Clin Oncol 25: 587-595, 2007.

21. Inukai M, Toyooka S, Ito $\mathrm{S}$, et al: Presence of epidermal growth factor receptor gene T790M mutation as a minor clone in non-small cell lung cancer. Cancer Res 66: 7854-7858, 2006.

22. Sequist LV, Martins RG, Spigel D, et al: First-line gefitinib in patients with advanced non-small-cell lung cancer harboring somatic EGFR mutations. J Clin Oncol 26: 2442-2449, 2008.

23. Toyooka S, Kiura K and Mitsudomi T: EGFR mutation and response of lung cancer to gefitinib. N Engl J Med 352: 2136, 2005.

24. Bell DW, Gore I, Okimoto RA, et al: Inherited susceptibility to lung cancer may be associated with the T790M drug resistance mutation in EGFR. Nat Genet 37: 1315-1316, 2005.

25. Koizumi F, Shimoyama T, Taguchi F, Saijo N and Nishio K: Establishment of a human non-small cell lung cancer cell line resistant to gefitinib. Int J Cance 116: 36-44, 2005.

26. Engelman JA, Mukohara T, Zejnullahu K, et al: Allelic dilution obscures detection of a biologically significant resistance mutation in EGFR-amplified lung cancer. J Clin Invest 116: 2695-2706, 2006.

27. Engelman JA, Zejnullahu K, Mitsudomi T, et al: MET amplification leads to gefitinib resistance in lung cancer by activating ERBB3 signaling. Science 316: 1039-1043, 2007.

28. Adjei AA: K-ras as a target for lung cancer therapy. J Thorac Oncol 3: S160-S163, 2008.

29. Suda K, Tomizawa K, Fujii M, et al: Epithelial to mesenchymal transition in an epidermal growth factor receptor-mutant lung cancer cell line with acquired resistance to erlotinib. J Thorac Oncol 6: 1152-1161, 2011.

30. Rho JK, Choi YJ, Lee JK, et al: Epithelial to mesenchymal transition derived from repeated exposure to gefitinib determines the sensitivity to EGFR inhibitors in A549, a non-small cell lung cancer cell line. Lung Cancer 63: 219-226, 2009. 